\title{
Concentraciones de DDT/DDE y riesgo de hipospadias. Un estudio piloto de casos y controles
}

\begin{abstract}
Silvia Flores-Luévano, MD, M en $C_{\text {, }}^{(1)}$ Paulina Farías, MD,PhD, (2) Mauricio Hernández, PhD, (2) Patricia Romano-Riquer, M en $C$, ${ }^{(2)}$ Jean Philippe W eber, MD, PhD, ${ }^{(3)}$ Eric D ewailly, MD,PhD, Juan Cuevas-Alpuche, MD, ${ }^{(5)}$ Isabelle Romieu,MD, ScD, M PH. ${ }^{(2)}$
\end{abstract}

Flores-Luévano S, Farías $\mathrm{P}$, Hernández M, Romano-Riquer $\mathrm{P}$, Weber JP, Dewailly E, Cuevas-Alpuche J, Romieu I. Concentraciones de DDT/DDE y riesgo de hipospadias. Un estudio piloto de casos y controles. Salud Publica Mex 2003;45:431-438. El texto completo en inglés de este artículo está disponible en: http://www.insp.mx/salud/index.html

\section{Resumen}

Objetivo. Evaluar la asociación entre los niveles séricos maternos de DDT/DDE y el riesgo de hipospadias. Material y métodos Estudio de casos y controles, efectuado en la Ciudad de México durante el periodo 1997-1999, con 41 casos de hipospadias y 28 controles. La informacion obtenida por cuestionario y las muestras sanguíneas fueron evaluadas mediante modelos multivariados de regresión lo gística. Resultados Las concentraciones materna de DDT y DDE no mostraron asociación con el riesgo de hipospadias (RM 1.13; IC 95\% 0.24-5.29 y RM 0.48; IC 95\% 0.15-1.60, respectivamente). Los predictores encontrados fueron hijos de madres con mayor edad (RM 8.69; IC 95\% 1.69-44.9), y la actividad laboral durante el embarazo (RM, 4.68; IC 95\% 1.15-18.9). Conclusiones Dado que se cuenta con escasa información sobre los efectos endocrinos a diferentes concentraciones de DDT/DDE en humanos, la dosis pudiera ser un factor determinante sobre el tipo de efecto. El texto completo en inglés de este artículo está disponible en: http://www.insp.mx/salud/index.html

Palabras clave: hipospadia, plaguicidas organoclorados; DDT; DDE; México
Flores-Luévano S, Farías P, Hernández M, Romano-Riquer $\mathrm{P}$,

Weber JP, Dewailly E, Cuevas-Alpuche J, Romieu I.

DDT/DDE concentrations and risk of hypospadias.

A case-control pilot study.

Salud Publica Mex 2003;45:431-438.

The English version of this paper

is available at: http://www.insp.mx/salud/index.html

\begin{abstract}
A bstract
O bjective. To evaluate the association between maternal serum DDT/DDE levels and risk of hypospadias. Material and Methods. A case-control study in Mexico City was conducted during 1997-1999 among 41 subjects with hypospadias and 28 controls. Blood samples were obtained from study subjects. Information was obtained using a standardized questionnaire. Data were analyzed using multivariate logistic regression models. Results. Maternal DDT and DDE levels showed no association with hypospadias (OR 1.13; $95 \% \mathrm{Cl} 0.24-5.29$ and $\mathrm{OR}=0.48 ; 95 \% \mathrm{Cl}$ 0.15-1.60, respectively). Associated factors were older age of mothers (OR $8.69 ; 95 \% \mathrm{CI} 1.69-44.9)$, and working during pregnancy (O R 4.68; $95 \% \mathrm{Cl} 1.15-18.9$ ). Conclusions. Information about the endocrine effects of different levels of DDT/DDE in human subjects is scarce; dosage may be a determinant factor of the type of effect. The English version of this paper is available at: http://www.insp.mx/salud/index.html
\end{abstract}

Key words: hypospadias; organochlorine pesticides; DDT; DDE; Mexico

Estudio realizado con apoyo del National C enter for Environmental Health/ Centers for D iseases Control and Prevention,Atlanta, GA. Estados U nidos de América.

(1) Instituto Mexicano del Seguro Social, D elegación Ciudad Juárez, Chihuahua, México.

(2) Instituto N acional de Salud Pública, Centro de investigaciones en Salud Poblacional. Cuernavaca, Morelos, México.

(3) Centre de Toxicologie du Q uébec, $C$ anada.

(4) Centre de Sante Publique de Q uébec, Service Sante et Environnement, Q uébec, C anada.

(5) Instituto Nacional de Pediatría. México, DF, México.

Fecha de recibido: 25 de julio de 2002 - Fecha de aprobado: 22 de mayo de 2003

Solicitud de sobretiros: Dra. Silvia Flores Luévano. Instituto Mexicano del Seguro Social, HGZ N o.6 Servicio de Pediatría. A nillo Envolvente del Pronaf N 0. 3970, 32315 Ciudad Juárez, Chihuahua,México.

Correo electrónico: silflor@prodigy.net.mx 
a hipospadias es una malformación congénita en la cual el meato urinario se encuentra en situación anormal, en la parte ventral del pene, el escroto o el periné.

Reportes mundiales indican una elevada incidencia de hipospadias en países europeos ${ }^{1,2}$ y en los Estados Unidos de América (EUA), ${ }^{3}$ lo que se incrementó inexplicablemente hasta cinco veces en un periodo de 25 años, con una tasa aproximada de 8.2/1 000 nacidos vivos. ${ }^{4}$ En México no se conocen con precisión las cifras con que se presenta, sin embargo, las malformaciones congénitas actualmente representan la cuarta causa de morbilidad y la segunda de mortalidad en el periodo de 1991 a 1999 en niños menores de un año de edad. ${ }^{5}$ Aunque las causas pueden ser múltiples, se estima que en 65 a $70 \%$ de las malformaciones congénitas éstas permanecen desconocidas, algunas de las cuales pudieran estar relacionadas con agentes ambientales. $^{6}$

Las principales hipótesis para explicar su etiología incluyen defectos en la síntesis de testosterona, mutación de los receptores androgénicos y de algunas enzimas, administración de progesterona y la participación de disruptores endocrinos. ${ }^{7-9}$ La participación de los factores genéticos es reconocida sólo para los casos severos de hipospadias y, secundariamente, para síndromes genéticos complejos y alteraciones cromosómicas, incluyendo los síndromes de desvanecimiento testicular, el de insensibilidad a los andrógenos y el de deficiencia tipo 2 de la 5 - $\alpha$-reductasa..$^{10} \mathrm{Un}$ factor hereditario se ha postulado en la génesis de hipospadias relacionado con genes autosómicos y dominantes. Los niños con hipospadias tienen una recurrencia en los hermanos de $17 \%$, con un factor de heredabilidad de $57 \%$. 11,12

Recientemente se ha estudiado al (DDT) y sus metabolitos, como el (DDE) y otros, por su efecto en la inhibición de los receptores androgénicos. La evidencia se basa en estudios experimentales hechos en animales. Dichos estudios mostraron capacidad para atravesar la barrera placentaria induciendo a bajas dosis, disminución de la distancia anogenital y pezones supernumerarios y, en dosis medias o altas, hipospadias, criptorquidia y alteraciones ependimarias. ${ }^{13,14}$

Los primeros ensayos clínicos hechos en mujeres embarazadas para observar el efecto de los estrógenos (dietiletilbestrol) reportaron un incremento de presentación de anomalías del tracto genital en el grupo de tratamiento (33\%), comparado con el grupo control $(8 \%),{ }^{15}$ y se duplicó el efecto entre aquellas mujeres que tuvieron la exposición antes de la semana 11 de gestación. ${ }^{16}$ Diferentes estudios epidemiológicos han reportado la asociación entre el consumo de hormonales en las primeras semanas de gestación, edad ma- terna, infertilidad familiar, primiparidad, abortos, bajo peso al nacer y exposición ocupacional, con el desarrollo de hipospadias y de criptorquidia. ${ }^{11,17}$ Asimismo, otros estudios encontraron malformaciones congénitas que incluyen hipospadias en algunas poblaciones cercanas a basureros y granjas, sitios donde se desechan o utilizan plaguicidas. ${ }^{2,18}$

En México, durante la década de 1950, se emplearon alrededor de 1000 toneladas de DDT al año para uso agropecuario. Su utilización y producción tuvo su pico máximo en la década de 1960, durante la cual se llegó a producir, en 1968, más de 80000 toneladas al año; debido a las evidencias de toxicidad en animales y a su alta persistencia en el ambiente en 1970 se prohíbe su uso en EUA y Canadá. En México, en 1987, se crea la Comisión Intersecretarial para el Control del Proceso y Uso de Plaguicidas, Fertilizantes y Sustancias Tóxicas (Cicoplafest); para 1990 el uso de DDT fue restringido a campañas de salud pública y su uso se redujo a 3000 tons/año; en 1997 se anuncia un programa que plantea la reducción del uso de DDT en los siguientes cinco años en 80\% (600 tons/año), y se plantea su gradual disminución hasta el cese total para el año $2007 \cdot{ }^{19-22}$ Debido a 50 años de utilización, a su persistencia y acumulación en la cadena alimenticia, la dieta podría ser la mayor fuente de exposición entre la población general; sin embargo, otras vías de exposición pueden ser a través del aire y el agua.

En un estudio de casos y controles desarrollado en pacientes de cáncer de mama, en la Ciudad de México, se encontraron elevados niveles de DDE; la media en suero, entre los casos, fue de $24.2 \mu \mathrm{g} / \mathrm{lt}$, y entre los controles, de $17.5 \mu \mathrm{g} / \mathrm{lt} .{ }^{23}$ De acuerdo con los resultados obtenidos en otro estudio, en el que fueron observados los efectos antiandrogénicos en animales de experimentación ( $63.3 \mathrm{ppb}){ }_{1}^{24}$ entre 3 y $5 \%$ de las mujeres que viven en la Ciudad de México están expuestas a los niveles de exposición.

En los últimos 50 años se han incrementado las anomalías del semen, el cáncer testicular y la criptorquidia, conjuntamente con hipospadias, defectos ligados probablemente a un origen común durante la embriogénesis. ${ }^{25}$ Este rápido aumento en las tasas, y la falta de evidencia de factores directamente relacionados con la génesis de la enfermedad nos llevan a considerar la participación ambiental como la responsable. Nuestro objetivo consiste en evaluar la concentración materna de DDT y DDE en su relación con el riesgo de hipospadias.

\section{Material y métodos}

Entre diciembre de 1997 y enero de 1999 se realizó un estudio de casos y controles en el Hospital Infantil de 
México y en el Instituto Nacional de Pediatría (hospitales de tercer nivel de atención, que concentran más de $90 \%$ de los casos que en el Valle de México no tienen derecho a seguridad social). Se identificaron 41 casos de hipospadias provenientes de la consulta de urología pediátrica en menores de 18 años de edad, mediante la revisión exhaustiva de expedientes clínicos, verificando el diagnóstico presuntivo o la fecha de cirugía. Se contactó a los padres de los niños, mediante cita telefónica o visita domiciliaria, y se les hizo una invitación por escrito. Además, se seleccionaron controles, de la misma fuente de obtención de los casos, mediante un tamizaje que reunió información acerca de la exposición y otras variables que permitieron conocer el estado de elegilibilidad de los participantes, pareados por grupos de edad ( \pm 2 años). Con el propósito de evitar o minimizar sesgos de información se consideró de utilidad tener un grupo control con alguna malformación congénita, y otro grupo, sin malformaciones. Al finalizar el tiempo y financiamiento del estudio, y al revisar aquellos participantes elegibles, solamente se reclutaron 28 controles con las características mencionadas. Se obtuvieron así dos grupos control: a) menores de 18 años de edad, con diagnóstico de microtia $(n=13), y$ b) menores de 18 años de edad, con patología no relacionadas con plaguicidas, como enuresis, plastia umbilical y alergia alimentaria $(n=15)$. Fueron excluidos niños procedentes de embarazos múltiples o con complicaciones maternas al final del embarazo, como toxemia o diabetes. Cuando los padres rehusaron participar se les solicitó que contestaran el mismo cuestionario corto aplicado a los casos.

\section{Medición de la exposición}

En ambos grupos se determinaron los niveles maternos en plasma de p’p`DDT, p`p ' DDE, así como otros compuestos organoclorados. Las concentraciones de estos compuestos han mostrado mantenerse estables durante tiempo prolongado, por lo cual es posible medir los niveles séricos años después del embarazo, como un adecuado reflejo de la exposición durante la vida fetal.

Las mediciones de compuestos organoclorados se hicieron en el centro de Toxicología de Québec por medio de un cromatógrafo de gases (Hewlett-Packard, 5890, Series II), equipado con columnas de capilares dobles y dos detectores de captura de electrones. Los límites de detección fueron de 0.02 a $0.03 \mu \mathrm{g} / \mathrm{l}$, y con un tiempo de recuperación de $90-103 \%$. La precisión entre días fue de $3.4 \%$ para DDE.

Las concentraciones de lípidos séricos fueron estimadas del total de colesterol y triglicéridos. El co- lesterol libre fue calculado como $0.27 \times$ colesterol total, de acuerdo con los parámetros de Cheek y Wease. ${ }^{26} \mathrm{La}$ estimación de los lípidos totales se hizo por adición del componente lipídico con la siguiente fórmula: ${ }^{27}$ lípidos totales séricos $=1.677$ (colesterol total-colesterol libre) + colesterol libre + triglicéridos +0.623 . La expresión de las concentraciones de organoclorados fue calculada basándonos en el peso de un lípido, lo cual garantiza la comparación de los resultados con muestras tomadas en ayuno o posprandialmente.

\section{Instrumentos de recolección de la información}

Se aplicó a los padres un cuestionario estandarizado para investigar las fuentes de exposición a compuestos organoclorados, el que incluyó variables sociodemográficas, enfermedades hereditarias e historia ginecológica, lo que incluyó abortos, exposición ocupacional, edad materna al nacimiento, paridad, exposición a medicamentos durante el primer trimestre del embarazo e historia de lactancia. Simultáneamente, se aplicó un cuestionario breve sobre actividad ocupacional.

\section{Muestra sanguínea}

Por personal especializado se tomó una muestra de 10 $\mathrm{ml}$ de sangre venosa del brazo de la madre; se empleó material nuevo, estéril y desechable. Las muestras de sangre fueron centrifugadas a $2000 \mathrm{rpm}$ durante 10 minutos. El plasma fue separado utilizando una bombilla pequeña y pipetas de vidrio, desechables Pasteur, previamente tratadas a $450{ }^{\circ} \mathrm{C}$. Después de su separación, el plasma se almacenó en tubos de vidrio de Wheaton de $6 \mathrm{ml}$, con tapa de teflón. Las muestras se mantuvieron en congelación a $-20{ }^{\circ} \mathrm{C}$ hasta ser analizadas. Se tuvo especial cuidado en que ninguno de los materiales o la muestra estuvieran en contacto con plástico, y se utilizaron guantes de nitrilo para el procedimiento.

\section{A nálisis estadístico}

Se realizó un análisis exploratorio para describir las características generales de la población de estudio y, posteriormente, se utilizaron análisis crudos y ajustados mediante regresión logística no condicionada, usando el paquete estadístico STATA V-6. ${ }^{*}$ Se emplea-

\footnotetext{
* STATA CORPORATION, 702 Drive East, Collage Station, TX 77840:
} http//www.stata.com 
ron también razones de momios y sus intervalos de confianza de $95 \%$. En los modelos multivariados las covariables de ajuste fueron la actividad laboral durante el embarazo, lactancia, edad de la madre, ocupación con plaguicidas y escolaridad. La evaluación global del modelo se logró a través de la prueba de bondad de ajuste de Hosmer-Lemeshow. Finalmente, se realizó una regresión lineal múltiple, para evaluar aquellas variables que predicen las concentraciones sanguíneas de DDT y DDE.

\section{Resultados}

Se obtuvieron 44 casos de hipospadias, diagnosticados entre las edades de 2 y 17 años. Se seleccionó a 28 controles que acudieron a la consulta de urología por motivo no relacionado con hipospadias o criptorquidia. El cuadro I muestra las principales características de la población. Los casos fueron, en promedio, menores de edad, respecto a los controles (media de 5.2 y 8.2 años, respectivamente) en forma significativa ( $p=0.001)$. Otras características no mostraron diferencias significativas en el entorno de los padres, en relación con sus edades, ingreso mensual, número de hijos y meses de lactancia materna. El cuadro II ofrece otras características de interés: antecedentes obstétricos, como problemas para embarazarse, abortos previos y padecimientos durante el embarazo, que resultaron más prevalentes entre los controles. De manera general, el consumo de medicamentos durante el embarazo fue más frecuente entre los controles. Las malformaciones congénitas en la familia fueron más prevalentes en los casos (22\%) que en los controles (18\%). Como ante-

\section{Cuadro I \\ DistribuCIÓn de CARACTERísticas SELECCIONADAS entre CASOS Y CONTROLES. MéXICO, 1997-1999}

\begin{tabular}{|c|c|c|c|c|c|}
\hline \multirow[b]{2}{*}{ Factores } & \multicolumn{2}{|c|}{$\begin{array}{l}\text { Casos } \\
\mathrm{n}=41\end{array}$} & \multicolumn{2}{|c|}{$\begin{array}{c}\text { Controles } \\
n=28\end{array}$} & \\
\hline & Media & $D E^{*}$ & Media & $D E^{*}$ & \\
\hline Edad (meses) & 63.0 & 48.4 & 99.1 & 41.7 & \\
\hline Edad materna (años) & 26 & 5.6 & 25 & 5.1 & \\
\hline Edad paterna (años) & 26 & 5.4 & 29 & 7.0 & \\
\hline Habitantes por casa & 5.1 & 2.0 & 5.2 & 1.8 & \\
\hline Personas en la casa con salario & 1.4 & 0.6 & 1.3 & 0.4 & \\
\hline Ingreso familiar mensual (pesos) & 1990 & 2368 & 2609 & 2609 & \\
\hline Número de hijos & 2.4 & 1.3 & 2.3 & 1.0 & \\
\hline actancia total (meses) & 14.6 & 16.6 & 11.9 & 11.9 & \\
\hline
\end{tabular}

* DE: D esviación estándar

\section{Cuadro II \\ DistRIBUCIÓN DE CARACTERISTICAS OBSTÉTRICAS, FAMILIARES Y LABORALES, ENTRE CASOS Y CONTROLES. MÉXICO, 1997-1999}

\begin{tabular}{llll} 
Factores & $\begin{array}{ccc}\text { Casos } \\
(n=41)\end{array}$ & $\begin{array}{c}\text { Controles } \\
(n=28)\end{array}$ & Valor* \\
\cline { 2 - 3 } & $\frac{n}{n} \%$ &
\end{tabular}

O bstétricos

\begin{tabular}{lllllll} 
Dificultad para embarazarse & & 3 & 7 & 4 & 14 & 0.34 \\
\hline
\end{tabular}

\begin{tabular}{llllll} 
Abortos previos & 6 & 15 & 8 & 28 & 0.15 \\
\hline
\end{tabular}

\begin{tabular}{llllll} 
A borto previo al nacimiento & 3 & 7 & 3 & 10 & 0.62 \\
\hline
\end{tabular}

$\begin{array}{llllll}\text { Fiebre en el embarazo } & 3 & 7 & 2 & 7 & 0.97\end{array}$

$\begin{array}{llllll}\text { Enfermedades en el embarazo } & 15 & 36 & 13 & 46 & 0.41\end{array}$

\begin{tabular}{llllll} 
Infecciones en el embarazo & 9 & 22 & 6 & 21 & 0.95 \\
\hline
\end{tabular}

$\begin{array}{llllll}\text { Lactancia } & 32 & 78 & 26 & 93 & 0.09\end{array}$

$\begin{array}{llllll}\text { Medicamentos en el embarazo } & 23 & 56 & 20 & 71 & 0.19\end{array}$

$\begin{array}{llllll}\text { Antibióticos } & 8 & 19 & 4 & 14 & 0.57\end{array}$

\begin{tabular}{llllll} 
Analgésicos & 4 & 10 & 3 & 11 & 0.89 \\
\hline Vitaminas
\end{tabular}

\begin{tabular}{llllll} 
Vitaminas & 4 & 10 & 7 & 25 & 0.08 \\
\hline
\end{tabular}

\begin{tabular}{llllll} 
Hormonales & 1 & 2 & 3 & 11 & 0.14 \\
\hline
\end{tabular}

\begin{tabular}{llllll} 
No especificados & 6 & 14 & 3 & 11 & 0.63 \\
\hline
\end{tabular}

$\begin{array}{llllll}\text { Tabaquismo } & 4 & 10 & 5 & 18 & 0.32\end{array}$

Familiares

$\begin{array}{llllll}\text { Malformaciones congénitas } & 9 & 22 & 5 & 18 & 0.67\end{array}$

$\begin{array}{lllllll}\text { Problemas reproductivos } & 4 & 10 & 5 & 18 & 0.32\end{array}$

Laborales

\begin{tabular}{lrrrrr} 
Trabaja actualmente & 12 & 29 & 11 & 39 & 0.38 \\
\hline Ama de casa & 29 & 71 & 17 & 61 & \\
\hline Relacionados con plaguicidas & 11 & 27 & 11 & 40 & 0.27 \\
\hline Trabajó en el embarazo & 15 & 37 & 5 & 18 & 0.09 \\
\hline $\begin{array}{l}\text { Trabajó con plaguicidas durante el embarazo } \\
0.85\end{array}$ & 5 & 12 & 3 & 11 \\
& & & & &
\end{tabular}

Escolaridad

\begin{tabular}{lrrrrr} 
Primaria o menos & 14 & 34 & 8 & 29 & 0.62 \\
\hline Secundaria o más & 27 & 66 & 20 & 71 &
\end{tabular}

* Prueba de $\chi^{2}$

cedente laboral de importancia cabe señalar que la actividad laboral durante el embarazo prevaleció entre los casos ( $37 \%$ vs. $17 \%$, respectivamente), sin ser la diferencia estadísticamente significativa.

El cuadro III muestra las concentraciones séricas de compuestos organoclorados entre ambos grupos. En promedio, la concentración de organoclorados fue menor en los casos que en los controles, a excepción del $p^{\prime} p^{\prime}$ DDT. Las diferencias de concentración sérica 


\section{Cuadro III \\ Concentraciones de plaguicidas organoclorados MATERNOS DE NIÑOS CON HIPOSPADIAS y sus controles. México, 1997-1999}

\begin{tabular}{|c|c|c|c|c|c|}
\hline \multirow[b]{2}{*}{ Tipo de organoclorado } & \multicolumn{2}{|c|}{ Casos } & \multicolumn{3}{|c|}{ Controles } \\
\hline & Media $(\mathrm{DE})^{*}$ & $\begin{array}{l}\text { Percentil } \\
10-90\end{array}$ & Media & $(\mathrm{DE})^{*}$ & $\begin{array}{r}\text { Percentil } \\
10-90\end{array}$ \\
\hline $1 \beta-B H C^{\ddagger}(\mu \mathrm{g} / \mathrm{lt})$ & $0.36 \quad(0.29)$ & $0.15-1.03$ & 0.56 & $(0.62)$ & $0.11-0.62$ \\
\hline $2 \beta$-BHC ( $\mu g / g$ líp) & $0.06 \quad(0.04)$ & $0.03-.015$ & 0.08 & $(0.04)$ & $0.02-0.12$ \\
\hline $3 p^{\prime} p^{\prime} D D T^{\S}(\mu g / t t)$ & $0.50(1.41)$ & $0.05-2.0$ & 0.38 & (0.73) & $0.05-0.92$ \\
\hline 4 p'p'DDT ( $\mu g / g$ líp) & $0.08 \quad(0.22)$ & $0.01-0.12$ & 0.05 & (0.10) & $0.01-0.13$ \\
\hline $5 p^{\prime} p^{\prime} D D E^{\#}(\mu g / / t)$ & $7.36 \quad(9.3)$ & $2.5-21.0$ & 9.50 & $(10.5)$ & $1.6-12.0$ \\
\hline $6 p^{\prime} p^{\prime} D D E(\mu g / g$ líp) & $1.2 \quad(1.69)$ & $0.39-3.44$ & 1.4 & $(1.32)$ & $0.29-2.14$ \\
\hline \multicolumn{6}{|c|}{$*(D E)=$ D esviación estándar } \\
\hline \multicolumn{6}{|c|}{$\ddagger \beta$-BHC = b-isómero de hexaclorobenceno } \\
\hline \multicolumn{6}{|c|}{$\begin{array}{l}\S p, p^{\prime} D D T=1,1,1 \text {-tricloro-2,2 bis ( } p \text {-clorofenil) etano } \\
\# p, p^{\prime} D D E=1,1, \text {-dicloro-2,2 bis ( } p \text {-clorofenil) etileno }\end{array}$} \\
\hline
\end{tabular}

de los compuestos organoclorados entre casos y controles no fueron estadísticamente significativas.

El cuadro IV presenta los resultados de la regresión logística. En el modelo multivariado la concentración de DDT no mostró asociación con el riesgo de hipospadias (RM, 1.13; IC 95\%, 0.24-5.29). De igual manera, las concentraciones de DDE no estuvieron relacionadas con el desarrollo de hipospadias (RM, 0.48; IC 95\%, 0.15-1.60). La escolaridad fue otra variable que no mostró asociación cuando los padres estudiaron secundaria o más (RM, 2.32; IC 95\%, 0.37-14.6). Los hijos de padres con mayor edad (entre 28 y 50 años) tuvieron menor riesgo de hipospadias, al compararlos con los hijos de padres más jóvenes (RM. 0.11; IC 95\%, 0.02-0.47), mientras que los hijos de madres con mayor edad tuvieron un riesgo nueve veces de padecer hipospadias, al compararlas con hijos de madres menores de 22 años de edad (RM, 8.69; IC 95\%, 1.69-44.9). Las malformaciones congénitas en la familia no estuvieron relacionadas con hipospadias (RM, 1.19; IC 95\%, 0.25-5.65). La actividad laboral durante el embarazo mostró un riesgo cinco veces mayor de hipospadias cuando se comparó con mujeres que no trabajaron durante el embarazo (RM, 4.68; IC 95\%, 1.15-18.9). El antecedente de abortos previos (RM, 1.27; IC 95\%, 0.169.74) y lactancia materna sí vs no (RM, 3.42; IC 95\%, 0.53-21.8) no estuvieron asociados con el desarrollo de hipospadias.

Los datos del modelo de regresión se ajustaron adecuadamente a las pruebas finales y se encontró una bondad de ajuste con una $p=0.25$ y un valor predictivo de $79 \%$. Los residuos se ajustaron a la curva de distribución estudentizada.

\section{Cuadro IV \\ Razones de MOMIOS E INTERVALOS DE CONFIANZA EN 41 CASOS DE HIPOSPADIAS Y 28 CONTROLES. Méxıco, 1997-1999}

Factor Casos Controles $\quad \mathrm{RM}^{\dagger} \quad$ IC $95 \% \quad \mathrm{RM}^{\ddagger} \quad$ IC $95 \%$

Concentración de DDT

(mg/g líp)

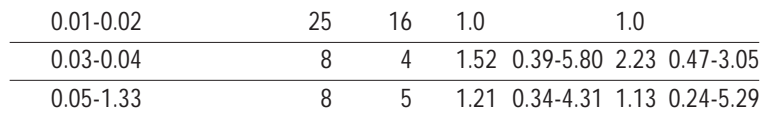

Concentración de DDE

( $\mathrm{mg} / \mathrm{g}$ líp)

\begin{tabular}{lllllll}
$0.16-0.84$ & 24 & 11 & 1.0 & 1.0 & \\
\hline $0.85-9.58$ & 17 & 17 & 0.45 & $0.17-1.22$ & 0.48 & $0.15-1.60$
\end{tabular}

Escolaridad

\begin{tabular}{|c|c|c|c|c|c|}
\hline Al menos primaria & 14 & 8 & 1.0 & & 1.0 \\
\hline Secundaria o más & 27 & 20 & 0.77 & $0.27-2.19$ & $2.32 \quad 0.37-14.6$ \\
\hline \multicolumn{6}{|l|}{ Edad del padre (años) } \\
\hline $16-27$ & 23 & 10 & 1.0 & & 1.0 \\
\hline $28-50$ & 13 & 17 & 0.33 & $0.11-0.93$ & $0.11 \quad 0.02-0.47$ \\
\hline
\end{tabular}

Edad de la madre (años)

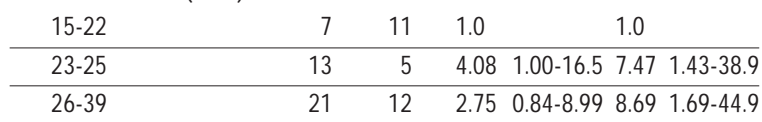

Malformaciones congénitas en la familia

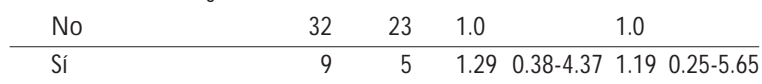

Laboró durante el embarazo

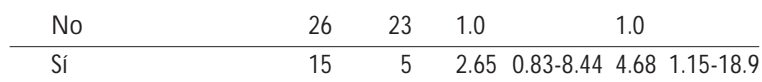

Abortos previos

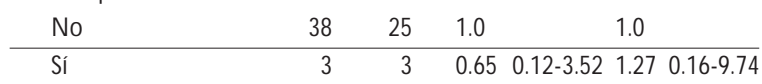

Lactancias

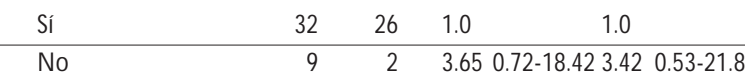

† Modelos univariados. RM (razón de momios)

* Modelo multivariado incluyendo las siguientes variables: edad de la madre, edad del padre, lactancia y actividad laboral durante el embarazo

Analizados en el modelo univariado se encontró al DDT como al mayor predictor de las concentraciones séricas de DDE, lo que explica hasta $31 \%$ de la varianza total, seguido por la edad de la madre, con $11 \%$, y la escolaridad con 1\% (datos no mostrados). 
Adicionalmente, se evaluaron en un modelo multivariado los potenciales predictores de concentraciones séricas de DDE, y los más importantes fueron la concentración de DDT, la edad y escolaridad $\left(r^{2}=0.70\right)$. El numero de hijos mostró una débil asociación (cuadro V), mientras que los principales predictores para concentraciones de DDT fueron la escolaridad y la edad materna $(\mathrm{r} 2=0.64)$.

\section{Discusión}

Los resultados no muestran diferencias en las concentraciones maternas de DDT y DDE entre los casos y los controles, así como tampoco hubo asociación con el riesgo de hipospadias.

Hasta donde sabemos, no se han publicado estudios relacionados con hipospadias y mediciones de organoclorados; sin embargo, hay determinaciones previas de organoclorados en dos estudios en México hechos con pacientes de cáncer de mama, los cuales reportaron una concentración media de DDE sérico en los casos de $24.2 \mu \mathrm{g} / \mathrm{lt}$, y $4.75 \mu \mathrm{g} / \mathrm{lt}$, respectivamente, en comparación con $7.36 \mu \mathrm{g} / \mathrm{lt}$ en este estudio; mientras que en los controles fue de $17.5 \mu \mathrm{g} / \mathrm{lt} 4.07 \mu \mathrm{g} / \mathrm{lt}$ en el mismo orden, comparado con $9.50 \mu \mathrm{g} / \mathrm{lt}$ de nuestro estudio, ${ }^{23,24}$ es conveniente mencionar que las determinaciones de las concentraciones de organoclorados

\section{Cuadro V \\ Predictores de concentraciones séricas DE DDE.* MÉXICO, 1997-1999}

\begin{tabular}{|c|c|c|c|}
\hline Factor & Beta $^{\ddagger}$ & Valor de $p$ & IC $95 \%$ \\
\hline Edad & 0.042 & 0.00 & $0.02,0.06$ \\
\hline \multicolumn{4}{|l|}{ Escolaridad } \\
\hline Secundaria o más & 0.413 & 0.00 & $0.11,0.70$ \\
\hline DDT sérico & 0.574 & 0.00 & $0.47,0.68$ \\
\hline \multicolumn{4}{|l|}{0 cupación relacionada } \\
\hline Con plaguicidas ${ }^{\#}$ & 0.067 & 0.59 & $-0.18,0.32$ \\
\hline $\mathrm{N}$ úmero de hijos & -0.12 & 0.05 & $-0.24,0.00$ \\
\hline \multicolumn{4}{|c|}{$\begin{array}{l}\text { * DDE= logaritmo de } 1,1 \text { diclo,2,2difeniletileno sérico ajustado a niveles } \\
\text { de lípidos totales } \\
\text { ₹ Modelo multivariado incluyendo las variables: edad, escolaridad, niveles } \\
\text { de DDT sérico, ocupación relacionada con plaguicidas y paridad } \\
\text { \& Categoría de referencia: primaria o menos } \\
\text { \# Categoría de referencia: no tener ocupación relacionada con plaguici- } \\
\text { das }\end{array}$} \\
\hline
\end{tabular}

en el primer estudio referido de cáncer de mama ${ }^{23}$ se efectuaron en el mismo laboratorio que en el del presente estudio. Considerando las concentraciones de DDE como el principal metabolito del DDT acumulado en el tejido adiposo, la edad es un factor importante para su evolución. En los estudios descritos la media de edad de los casos fue de 52 años, mientras que para los controles, de 49. Nuestro estudio muestra una media de edad inferior a estas cifras (25 y 26 años, respectivamente) lo que pudiera ser una razón que explique las diferencias encontradas.

Uno de los factores que puede estar involucrado es la edad de los padres; no obstante, la relación entre la edad materna y el riesgo de hipospadias no es consistente; algunos estudios no encontraron asociación con la edad materna; ${ }^{17,28}$ sin embargo, nuestros resultados sugieren un vínculo de la edad con el riesgo de hipospadias, lo cual está de acuerdo con recientes reportes, ${ }^{29}$ en los que se ha mostrado un incremento en el riesgo de hipospadias con mayor edad materna, además de mayor severidad de los casos, pues se encontró que $50 \%$ de los niños con hipospadias severas se presentaron en mujeres mayores de 35 años de edad. Una explicación es que la primiparidad como factor de riesgo podría estar asociada con el cambio en la edad de inicio de la paridad, ya que en los últimos 20 años el promedio es de los 30 e incluso más años de edad en los países desarrollados. Contrariamente, la edad paterna parece estar asociada inversamente al riesgo de hipospadias. Aunque no hay evidencia reportada, nuestros resultados sugieren esta posibilidad, que deberá evaluarse en un estudio con mayor poder estadístico.

Exposiciones durante tiempo prolongado a pequenas cantidades de p' p' DDT podrían dar lugar a acumulaciones mayores en tejido adiposo. Los plaguicidas organoclorados son excretados en la leche materna, y sus concentraciones están fuertemente correlacionadas con el contenido de grasa de la leche misma y con la edad materna. Por lo tanto, es el tiempo de exposición durante la vida de la mujer, más que la exposición durante el embarazo, lo que determina el nivel de exposición del feto, y del lactante alimentado con leche materna. ${ }^{30}$ Además, se ha encontrado que mujeres que han tenido óbitos o partos prematuros excretan mayor cantidad de p'p' DDT en su leche que aquellas que llegan al final de la gestación. ${ }^{31,32}$

Haber laborado durante el embarazo reveló ser un factor de riesgo importante en la presencia de hipospadias; sin embargo, cuando se exploró la asociación en las mujeres cuyas ocupaciones estuvieron relacionadas con plaguicidas (jardinería, agricultura, manejo o venta de plaguicidas) no se encontró un mayor 
riesgo, ni diferencias en los niveles de DDE séricos, respecto de otros tipos de empleo. Se requiere evaluar otros factores que podrían estar asociados con exposiciones laborales como riesgos ergonómicos y exposiciones a otros químicos. Esto es consistente con otros estudios en los cuales se encontró incremento en el riesgo de criptorquidia, pero no de hipospadias en hijos de las madres que laboran en granjas o en jardinería. ${ }^{33}$

Otros factores relacionados con el riesgo de hipospadias como abortos previos, paridad, malformaciones congénitas, o el consumo de hormonales durante la gestación no mostraron estar asociados en nuestro estudio a pesar de haber sido ya reportados. ${ }^{17,28}$

Es importante considerar dentro de las limitaciones del estudio que, por tratarse de uno de casos y controles, podría presentarse dificultad para establecer una medida precisa de la exposición durante el embarazo y la presencia de la enfermedad; no obstante que las concentraciones de los plaguicidas organoclorados han mostrado mantenerse estables durante tiempo prolongado, es posible medir los niveles séricos años después del embarazo, y esta medición puede tenerse como un adecuado reflejo de la exposición durante la vida fetal. Debido al efecto acumulado de estos plaguicidas es sustancial considerar que no hubo diferencias en la media de edad entre los grupos de estudio.

Otra limitación sobresaliente de este estudio es el pequeño tamaño de la muestra. Debido a la baja prevalencia de la enfermedad se deberá considerar, en el futuro, el desarrollo de estudios multicéntricos que permitan obtener un mayor número de sujetos de estudio. Las estimaciones realizadas para encontrar asociación con el DDT/DDE pudieron haber sido afectadas principalmente por este hecho; por ejemplo, el poder estadístico para estimar la asociación con DDT se elaboró con una potencia de 33\%, mientras que para DDE fue de $20 \%$. La amplitud del rango de poder estadístico fue desde $98 \%$, para edad materna, hasta un mínimo de $5 \%$ al analizar abortos y otras malformaciones congénitas.

Los estudios de malformaciones congénitas, como el aquí reportado, son más susceptibles de presentar sesgos de información; por ejemplo, es probable que la madre recuerde con mayor detalle y exactitud las exposiciones pasadas tratándose de los casos; por lo tanto, se consideró de utilidad tener un grupo control con alguna malformación congénita diferente a la del motivo del estudio, y otro grupo sin malformaciones; sin embargo se utilizó un marcador biológico de exposición no afectado por el recuerdo de los participantes.

Una gran parte de los estudios publicados con relación a malformaciones congénitas y plaguicidas se han llevado a cabo en personas ocupacionalmente expuestas, sin embargo, la mayor dificultad ha sido medir la exposición. Algunos autores han usado cuestionarios para estimarla o la han asignado de acuerdo con el perfil laboral (tipo y horario en el puesto), ${ }_{1}^{34,35}$ lo que limita la capacidad de comparación entre los diferentes estudios. En nuestro estudio, pese a ser uno piloto, se contó con determinación de marcadores biológicos que minimizan los errores para clasificar la exposición. Este trabajo fue hecho en la población expuesta en mayor medida a factores ambientales, a plaguicidas, como consecuencia de la contaminación del aire, del agua o de los alimentos, a concentraciones bajas. Sin embargo, los niveles de exposición ocupacional de la población crónicamente expuesta, como residentes en zonas de aplicación de DDT para el control de vectores, pueden ser mucho mayores, como lo demuestran los valores encontrados entre habitantes, no expuestos ocupacionalmente, de una zona endémica de malaria (Tapachula, Chiapas), donde la media de las concentraciones de $\mathrm{p}^{\prime} \mathrm{p}^{\prime} \mathrm{DDE}$ en lípidos séricos fue de $77.9 \mathrm{mg} / \mathrm{kg}(17.0-177.2),{ }^{36}$ lo cual es más de 100 veces mayor a lo documentado en poblaciones expuestas a niveles ambientales basales.

Algunos plaguicidas organoclorados, se ha demostrado, actúan como xenoestrógenos. Asimismo, se ha reportado que el DDT es un potente inductor de la activación microsomal, lo que puede ocasionar un descenso de los andrógenos testiculares por incremento en su degradación. ${ }^{37}$ Otros estudios han documentado que, tanto el DDT como el PCB y los alquifenoles actúan ligándose a receptores estrogénicos. ${ }^{38}$ En estudios in vitro se ha reportado que el p'p' DDE actúa, tanto bloqueando la respuesta celular a la testosterona, como ligando receptores androgénicos. ${ }^{13}$ Por ello se sospecha que estos químicos presentes en el ambiente pueden estar relacionados con deterioro reproductivo en hombres y animales. La mayor exposición de la población general a estos disruptores endocrinos es a través de la dieta (plaguicida, conservadora, contaminante, material que utilizan para su empaque y almacenamiento); no obstante, otras fuentes y vías de exposición también pueden presentarse en el aire, en el agua, por la utilización de contaminantes caseros, y a través de exposición ocupacional. ${ }^{35}$

Puesto que se cuenta con escasa información sobre los efectos endocrinos a diferentes concentraciones de DDT/DDE en humanos, la dosis pudiera ser un factor determinante sobre el tipo de efecto, como se ha visto en estudios experimentales con animales en los que el defecto de hipospadias se presenta sólo con dosis medias o altas, por lo que consideramos que este estudio puede ser un punto de comparación con otros que se hagan con población expuesta a concentraciones mayores de plaguicidas. 


\section{Referencias}

1.Toppari J, Larsen JC, C hristiansen P, G iwercman A, G randjean P, Guillette LJ Jr et al. Male reproductive health and environmental xenoestrogens. Environ Health Perspect 1996;104 Suppl 4:741-803. 2. Matlai $P$, Beral V.Trends in congenital malformations of external genitalia. Lancet 1985;1(8420):108.

3. Center for Disease Control. Leading major congenital malformations among minority groups in the United States, 1981-1986. MMW R Morb Mortal W kly Rep 1988;37:17-24.

4. Paulozzi LJ, Erickson JD, Jackson RJ. Hypospadias trends in two US surveillance systems. Pediatrics 1997;100(5):831-834.

5. Dirección General de Estadística e Informática. Secretaría de Salud, México, 1999.

6.W ilson J.Teratogenic effects of environmental chemicals. Fed Proc 1997;36:1698-1703.

7. Silver R.W hat is the etiology of hypospadias? A review of recent research. D el Med J 2000;72(8):343-347.

8. Bals-Pratsch M, Schweikert HU, N ieschlag E.Androgen receptor disorder in three brothers with bifid prepenile scrotum and hypospadias. Acta Endocrinol (Copenh) 1990;123(3):271-276. 9. Hiort 0 , Klauber G, Cendron M, Sinnecker GH, Keim L, Schwinger E et al. Molecular characterization of the androgen receptor gene in boys with hypospadias. Eur J Pediatr 1994;153(5):317-321.

10. Boehmer AL, N ijman RJ, Lammers BA, D e C oninck SJ,Van-Hemel JO, Themmen AP et al. Etiological studies of severe or familial hypospadias. J U rol 2001;165(4):1246-1254.

11. Harris EL. Genetic epidemiology of hypospadias. Epidemiol Rev 1990;12:29-40.

12. Stoll C, Alembik Y, Roth MP, D ott B. Genetic and environmental factors in hypospadias. J Med Genet 1990;27(9):559-563.

13. Kelce W R, Stone CR, Laws SC, G ray LE, Kemppainen JA,W ilson EM. Persistent DDT metabolite $p, p^{\prime}-D D E$ is a potent androgen receptor antagonist. N ature 1995;375(6532):581-585.

14. Gray LE, $O$ stby J, Furr J,W olf C J, Lambright C , Parks L et al. Effects of environmental antiandrogens on reproductive development in experimental animals. Hum Reprod Update 2001;7(3):248-264.

15. Gill W B, Schumacher GB, Straus M, Schoenberg HW. Association of diethylestilbestrol exposure in utero with cryptorchidism, testicular hypoplasia and semen abnormalities. J U rol 1979(122):36-39. 16.W ilcox AJ, Baird DD, W einberg CR, Hornsby PP, Herbst AL. Fertility in men exposed prenatally to diethylstilbestrol. N Engl J Med 1995;332(21):1411-1416.

17. Sloth W I, Moller H, Kold TJ, Skakkebaek N E. Risk factors for cryptorchidism and hypospadias. J U rol 1999(161):1606-1609.

18. Kristensen P, Irgens LM, Andersen A, Bye AS, Sundheim L. Birth defects among offspring of N orwegian farmers, 1967-1991.

Epidemiology 1997:8(5):537-544.

19. Panamerican Health O rganization Status of Malaria Programs in the Americas.W ashington: PAHO, 1992.

20. Secretaría de Medio Ambiente, Recursos N aturales y Pesca. Lo que usted debe saber sobre DDT y su uso en el combate al paludismo en México.México, DF: Semarnap,1996.
21. Secretaría de Medio Ambiente, Recursos N aturales y Pesca. Lo que usted debe saber sobre DDT y uso en el combate al plaguicidas: México DF: Semarnap, 1996.

22. Albert LA. Persistent pesticides in Mexico. Rev Environ Contam Toxicol 1996;147:1-44

23. Romieu I, Hernández-Avila M, Lazcano-Ponce E,W eber JP, D ewailly E. Breast cancer, lactation history, and serum organochlorines. Am J Epidemiol 2000;152(4):363-370.

24. López-C arrillo L, Blair A, López-Cervantes M, C ebrián M, Rueda C, Reyes $R$ et al. Dichlorodiphenyltrichloroethane serum levels and breast cancer risk:A case-control study from Mexico. Cancer Res 1997;57(17):3728-3732.

25. Giwercman A, C arlsen E, Keiding N , Skakkebaek N E. Evidence for increasing incidence of abnormalities of the human testis:A review. Environ Health Perspect 1993;101 Suppl 2:65-71.

26. Cheek CS,W ease DF.A summation technique for serum total lipids: Comparison of methods. Clin Chem 1969;15:102-107.

27. Phillips D L, Pirkle JL, Burse VW, Bernert JT Jr, Henderson LO, N eedham LL. Chlorinated hydrocarbon levels in human serum: Effects of fasting and feeding. Arch Environ Contam Toxicol 1989;18(4):495-500. 28. A kre 0, Lipworth L, C nattingius S, Sparen P, Ekbom A. Risk factor patterns for cryptorchidism and hypospadias. Epidemiology 1999;10(4):364-369.

29. Fish H, Golden RJ, Libersen GL, Hyun GS, Madsen P, N ew MI et al. Maternal age as a risk factor for hypospadias. J U rol 2001;165:934-936. 30. Kelce W R, W ilson EM. Environmental antiandrogens: D evelopmental effects, molecular mechanisms, and clinical implications. J Mol Med 1997;75(3):198-207.

31. Siddiqui MK, Saxena MC. Placenta and milk as excretory routes of lipophilic pesticides in women. Hum Toxicol 1985;4(3):249-254. 32. N oren K. C ontemporary and retrospective investigations of human milk in the trend studies of organochlorine contaminants in Sweden. Sci Total Environ 1993:139-140:347-355

33. Weidner IS, Maller H, Jensen TK, Skakkebaek N E. Cryptorchidism and hypospadias in sons of gardeners and farmers. Environ Health Perspect 1998(106):793-796.

34. Engels LS, 0 'M eara ES, Sw hwartz SM. Maternal ocupation in agriculture and risk of adverse birth outcomes in W ashington State, 1980-1991. Am J Epidemiol 1995(141):S73.

35. Restrepo M, Muñoz N , D ay N , Parra JE, Hernández C, Blettner M et al. Birth defects among children born to a population occupationally exposed to pesticides in Colombia. Scand JW ork Environ Health 1990;16(4):239-246.

36. Ayotte $P, G$ iroux $S, D$ ewailly $E, H$ ernández-A vila $M$, Farías $P, D$ anis $R$ et al. DDT spraying for malaria control and reproductive function in Mexican men. Epidemiology 2001;12(3):366-367.

37. Bulger W H, Muccitelli RM, Kupfer D. Studies on the in vivo and in vitro estrogenic activities of methoxychlor and its metabolites. Role of hepatic mono-oxygenase in methoxychlor activation. Biochem Pharmacol 1978(27):2417-2423.

38. W hite R, Jobling S, Hoare SA, Sumpter JP, Parker MG. Environmentally persistent alkylphenolic compound are estrogenic. Endocrinology 1994(135):175-182. 